\title{
Precision Family Spirit: a pilot randomized implementation trial of a precision home visiting approach with families in Michigan - trial rationale and study protocol
}

Allison Ingalls 1 $^{*}$, Allison Barlow', Elizabeth Kushman², Amanda Leonard², Lisa Martin², Precision Family Spirit Study Team², Allison L. West ${ }^{3}$, Nicole Neault ${ }^{1}$ and Emily E. Haroz ${ }^{1}$

\begin{abstract}
Background: Home visiting is a well-supported strategy for addressing maternal and child health disparities. However, evidence-based models generally share implementation challenges at scale, including engagement and retention of families. Precision home visiting may address this issue. This paper describes the first known pilot randomized implementation trial of a precision home visiting approach vs. standard implementation. Primary aims are to: 1 ) explore the acceptability and feasibility of a precision approach to home visiting and 2) examine the difference between Standard Family Spirit and Precision Family Spirit on participants' program satisfaction, clienthome visitor relationship, goal alliance, and the impact of these factors on participant engagement and retention. Secondary aims are to explore potential differences on maternal behavioral and mental health outcomes and child development outcomes to inform sample size estimations for a fully powered study.

Methods: This is a pilot Hybrid Type 3 implementation trial. Four Michigan communities primarily serving the Native American families and already using Family Spirit were randomized by site to receive Standard Family Spirit or Precision Family Spirit. Participants include $N=60$ mothers at least 14 years of age (pregnant or with a newborn $<2$ months of age) currently enrolled in Family Spirit. Precision Family Spirit participants receive core lessons plus additional lessons based on needs identified at baseline and that emerge during the trial. Control mothers receive the standard sequence of Family Spirit lessons. Data is collected at baseline ( $<2$ months postpartum), and 2, 6, and 12 months postpartum. All Precision Family Spirit participants are invited to complete qualitative interviews at study midpoint and endpoint. All home visitors are invited to participate in focus groups between study midpoint and endpoint. Exploratory data analysis will assess feasibility, acceptability, client-home visitor relationship, retention, adherence, and potential differences in intervention outcomes.
\end{abstract}

(Continued on next page)

* Correspondence: aingalls@jhu.edu

${ }^{1}$ Center for American Indian Health, Department of International Health, Johns Hopkins Bloomberg School of Public Health, Baltimore, MD, USA

Full list of author information is available at the end of the article

(c) The Author(s). 2021 Open Access This article is licensed under a Creative Commons Attribution 4.0 International License, which permits use, sharing, adaptation, distribution and reproduction in any medium or format, as long as you give appropriate credit to the original author(s) and the source, provide a link to the Creative Commons licence, and indicate if changes were made. The images or other third party material in this article are included in the article's Creative Commons licence, unless indicated otherwise in a credit line to the material. If material is not included in the article's Creative Commons licence and your intended use is not permitted by statutory regulation or exceeds the permitted use, you will need to obtain permission directly from the copyright holder. To view a copy of this licence, visit http://creativecommons.org/licenses/by/4.0/ The Creative Commons Public Domain Dedication waiver (http://creativecommons.org/publicdomain/zero/1.0/) applies to the data made available in this article, unless otherwise stated in a credit line to the data. 
(Continued from previous page)

Discussion: This trial will provide new information about the acceptability and feasibility of precision home visiting and pilot data on program satisfaction, client-home visitor relationship, goal alliance, retention, and targeted maternal-child intervention outcomes. Findings will inform the design of a fully powered randomized implementation trial of precision vs. standard home visiting.

Trial registration: ClinicalTrials.gov \#NCT03975530; Registered on June 5, 2019

Keywords: Precision science, Precision public health, Implementation science, Hybrid design, American Indian, Pregnancy and childbirth, Randomized controlled trials, Home-visiting

\section{Key messages on feasibility}

- Prior to this pilot study, it is unclear whether comparing a modularized version of a home visiting program to standard practice home visiting would be feasible, and, if so, whether there would be detectable differences between the approaches.

- Results will inform whether and how to design a fully powered randomized controlled trial to test implementation effectiveness of a modularized version of home visiting to improve primary outcomes.

- The results of the study will inform 1) how to approach randomization, 2) data collection platforms to use, 3) sample size calculations, and 4) adaptations to the design and measures for a fully powered randomized controlled trial.

\section{Background}

Decades of research validate early childhood home visiting as an effective strategy to support families. Home visiting programs target a range of maternal and child outcomes, including promoting healthy growth and development, and reducing maternal and child behavioral and mental health disparities. In 2010, the United States Congress authorized $\$ 1.5$ billion to fund the Maternal, Infant, and Early Childhood Home Visiting (MIECHV) Program across the United States (US). MIECHV was reauthorized and funded at $\$ 400$ million per year for 5 years in early 2018. The Home Visiting Evidence of Effectiveness (HomVEE) review identified 21 home visiting models that meet the US Health and Human Services criteria for an evidence-based early childhood home visiting service delivery model. Of these, 19 are eligible to be used with MIECHV funds $[1,2]$.

Evidence-based home visiting programs being disseminated across the US struggle with retention of families. A recent report indicated only half of participating families across four home visiting models remained in the program at one year postpartum, despite these models expecting that families remain enrolled until at least 2 years postpartum [3]. Many home visiting models were designed to serve families over several years with regularly scheduled visits. While some shorter home visiting models have seen positive outcomes, little is known regarding optimal duration and dosage [4]. Moreover, the types of families being served and the diversity of issues and challenges they present suggest the need to tailor and adapt programs to better fit a wide range of needs [5]. To date, this tailoring has almost always relied solely on the judgement of an individual home visitor or, in some cases, is guided by the implementing organization without guidance from the model developer.

Recent qualitative research on high- and low-retention sites using one evidenced-based home visiting model showed that nurses in high-retention sites were more collaborative with families. They were more likely to adapt their program delivery to align with families' needs, compared to nurses in low-retention sites [6]. Based on these findings, Ingoldsby and colleagues (2013) developed an implementation strategy grounded in the principals of motivational interviewing to provide nurses with more explicit control over the visit schedule and content to families. When they compared retention rates for nurses trained in motivational interviewing to rates for nurses trained in the standard approach, preliminary findings showed retention rates were significantly better when nurses had more flexibility in service delivery [7]. It is important to note that authors recognized several other factors that may be influencing these positive findings. Nonetheless, improving implementation of programs at scale including achieving high participant retention, enhancing program content to address families' unique and complex needs, and increasing model fidelity is of key interest to researchers, implementing agencies, and other stakeholders [8].

From 2016 through 2018, the Johns Hopkins Center for American Indian Health (CAIH) conducted formative work to develop an implementation strategy for Family Spirit ${ }^{\circ}$ based on concepts of design modularity [9]. Design modularity aims to systematically tailor content to meet families' needs. Family Spirit is a federally endorsed, evidence-based model designed for, by, and with Native American communities [10-12]. The rationale for the development of a precision implementation 
strategy for Family Spirit, called Precision Family Spirit, was based on two factors. First, home visitors are faced with the complex task of applying knowledge gained from previous research studies to a diverse range of clients who may be substantially different from the populations from which this research was generated. This yields potential problems with fidelity. Second, based on studies indicating tailoring may help with retention $[6$, 7], we sought to use modularization as an implementation strategy to tailor Family Spirit while maintaining fidelity to the model [13].

The current study is a pilot Hybrid Type 3 [14] implementation trial of Precision Family Spirit. This hybrid design tests an implementation strategy while also gathering data on relevant outcomes. This pilot study will examine the acceptability, feasibility, and preliminary effects of a "precision approach" to home visiting. Our specific aims are to 1) explore the acceptability and feasibility of a precision approach to home visiting from the perspective of enrolled mothers and 2) examine the difference between Precision Family Spirit and Standard Family Spirit on program satisfaction, client-home visitor relationship, goal alliance, and retention. As part of the hybrid design, we will also monitor client level intervention effects on maternal self-efficacy, stress, and child social, emotional, and behavioral development.

\section{Methods/design}

\section{Trial design}

This study uses a two-arm pilot randomized Hybrid Type 3 design [14]. Four Family Spirit home visiting sites from the Inter-Tribal Council of Michigan (ITC of MI) were selected based on comparability and randomized using a 1:1 allocation to provide either Standard Family Spirit or Precision Family Spirit to their clients. Sites were matched based on annual volume of clients served and geographic similarity (i.e., urban vs. rural) (Fig. 1).

\section{Participants}

Our target population for recruitment is women who are either pregnant or have a baby no older than 2 months of age. All study participants must be at least 14 years of age and already enrolled in the Family Spirit program in their community. Since 2013, the CAIH and the ITC of MI have partnered on implementing the Family Spirit model across participating tribal communities. The ITC of MI has a strong infrastructure to support the incorporation of a research study into their existing home visiting programming.

\section{Participating communities}

Four ITC of MI sites are participating in this pilot trial, all located in the US and primarily serving Native
American families-two in the Upper Peninsula of Michigan (MI) and two in the Lower Peninsula of MI, one of which is an Urban Indian Organization (UIO). The ITC of MI was founded in 1968 to combine efforts of a few Native American tribal communities. Working collaboratively allowed these tribal communities to leverage resources and expand programs to enhance the health and well-being of tribal members. Today, the ITC of MI is a consortium of all 12 federally recognized tribes and 1 UIO in Michigan. Member tribes directly manage local program operations while ITC of MI provides technical assistance and administrative support, often serving as grantee on projects involving a consortium of tribes. To respect tribal sovereignty and their wishes, the participating Tribes' names have been left out of this paper.

Native American communities in the Upper Peninsula are rural and geographically isolated. While a sizeable portion of the population lives on reservation land in relative proximity to tribal services, many live off reservation across a wide area of multiple counties. Historical trauma and government policies which disrupted tribal communities' economic, social, and cultural structures and overall way of life have led to disparities across income, employment, health, and wellness compared to the Michigan general population. Likewise, Lower Peninsula tribal communities face socioeconomic and health disparities as a result of historical trauma and government policies. While Lower Peninsula communities are characterized as more semi-urban and urban as compared to those in the Upper Peninsula, their families are facing similar challenges. Thus, both Lower and Upper Peninsula communities have been using home visiting as a part of a larger strategy to strengthen families.

\section{Family Spirit Model}

The Family Spirit evidence-based home visiting model was designed and tested by the CAIH in partnership with tribal communities over a 20 -year period [10]. It is currently the largest, most rigorous, and only evidencebased home visiting program designed for pregnant and parenting Native American families. Family Spirit trains Native American community members to serve as home visitors who provide structured, culturally grounded education to families with children ages 0 to 3 . The goal is to produce healthier pregnancies and healthier future generations by providing education around pregnancy, parenting, child development, and life skills and connecting families with community resources. The model was designed for delivery by paraprofessionals but can also be delivered by professionals. The employment of paraprofessionals to deliver the program is more sustainable and builds capacity within communities that suffer low employment and poor access to health care and 


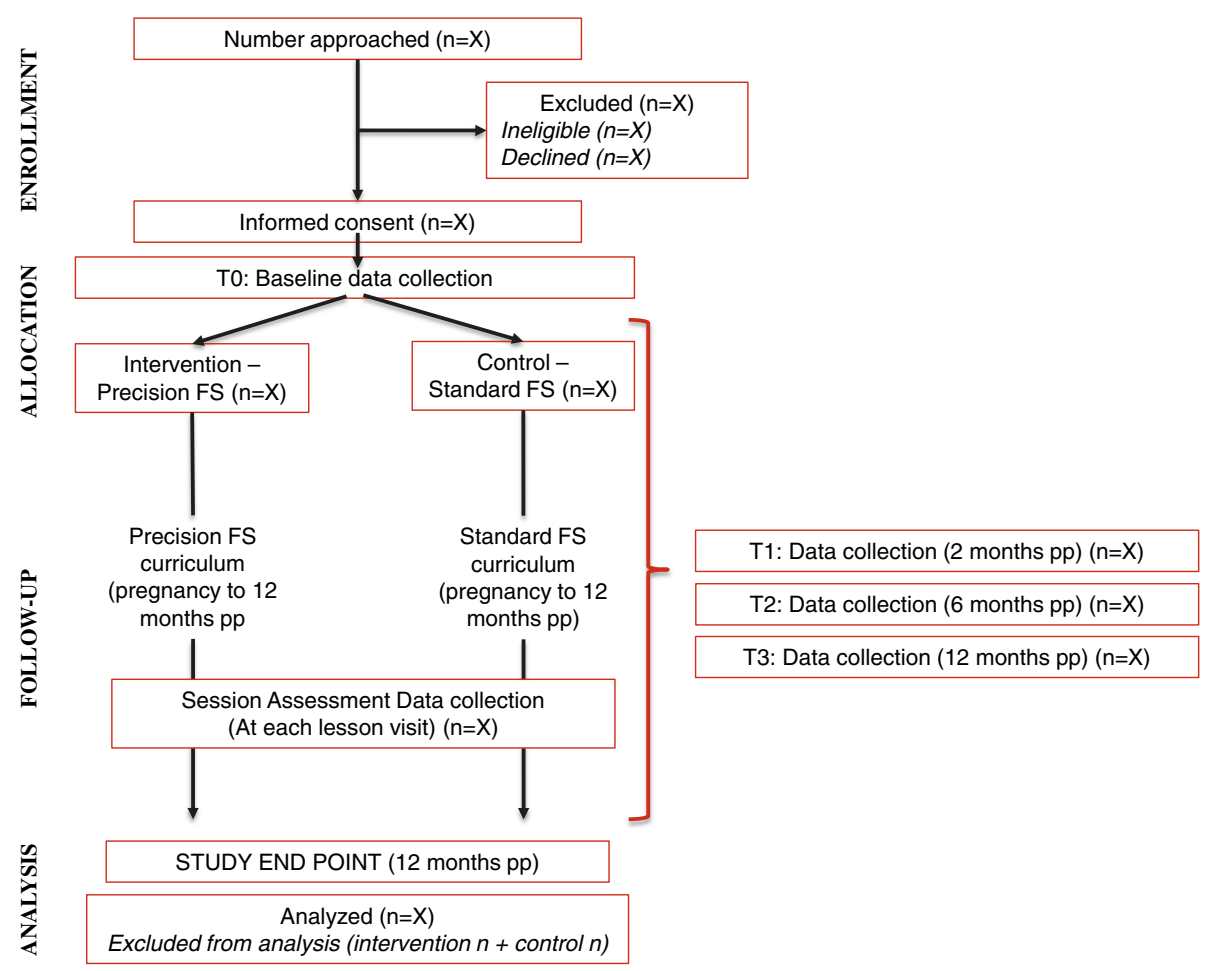

Fig. 1 Study design and data collection

education. It also allows for cultural match between home visitors and participants, as most professionals in the communities are non-Native.

Family Spirit utilizes a 63-lesson curriculum, which was designed through a community-based participatory process led by the CAIH in partnership with three Southwestern Native American tribal communities. The original curriculum was evaluated through three successive randomized controlled trials (RCTs), each one increasing in length and rigor and corroborating prior findings. In the third and largest RCT, all families in the intervention study arm received all Family Spirit lessons. Lesson visits typically lasted an hour and started out weekly and decreased in frequency over time, until they were being taught bimonthly from 2 to 3 years postpartum. Family Spirit has demonstrated the following impacts: increases in parenting knowledge $[10-12,15]$ and self-efficacy $[10,15]$; reductions in parenting stress $[12$, $15]$ and maternal psychosocial risks that could impede positive parenting, including substance use [15] and depression [11, 12, 15]; and improved children's social, emotional, and behavioral development $[10,12,15]$. Based on these findings, in 2013, Family Spirit received the highest ratings for an "evidence-based" program from the federal HomVEE review of home visiting program effectiveness and a 4.0 out of 4.0 for dissemination readiness from the National Registry for Evidence-Based Practices and Programs (NREPP). At present, over 130 tribal communities and 4 urban non-Native communities across 22 US states have been trained to deliver Family Spirit. The program has been adopted by nonNative communities due to its unique cultural strengths, use of paraprofessionals, and strong evidence of effectiveness. Family Spirit respects tribal data sovereignty and thus does not report nationally on retention of participating families. However, like other home visiting models being disseminated across the US, Family Spirit affiliates have shared challenges with retention and engagement of families for the duration of the program. A precision approach to Family Spirit provides promise to addressing this issue of implementation of an evidencebased home visiting program at scale.

\section{Recruitment}

The pilot study is recruiting $N=60$ women who meet the inclusion and exclusion criteria below:

\section{Inclusion criteria}

1. Women who are either pregnant or have a baby no older than 2 months old

2. At least 14 years of age at time of conception

3. Enrolled in Family Spirit services in the participating community 


\section{Exclusion criteria}

1. Inability to participate in full intervention or evaluation (e.g., planned move, scheduling conflicts, etc.)

Ambiguous cases based on unclear age at conception or unclear gestational age are reviewed by the Study Management Team before being deemed eligible for recruitment. Home visitors conduct informed consent procedures with all eligible participants. All participants must provide informed consent or assent to participate. Participants under age 18 must have parent/guardian consent. The first participant gave consent on June 25, 2019, and the first baseline assessment was completed on June 26 , 2019.

\section{Randomization}

Participating ITC of MI sites were randomized to use the Precision Family Spirit implementation strategy or Standard Family Spirit (control condition). Randomization occurred before recruitment began at the site level. The four sites were selected based on comparability of annual volume of clients served and geographic similarity (i.e., urban vs. rural). Once sites were matched, the study team flipped a coin to decide which sites would be assigned to each study arm. Participants are blind to whether they receive Standard Family Spirit or Precision Family Spirit as randomization occurs at the site-level. Thus, all participants at each site receive the same version of Family Spirit (standard or precision).

\section{Ethical considerations}

This study was reviewed and approved by the Johns Hopkins Bloomberg School of Public Health Institutional Review Board (JHBSPH IRB) (IRB00009315; Current protocol version: \#9, approved on September 8, 2020). Any protocol modifications are submitted as an amendment to the JHBSPH IRB. Supervisors then inform study staff and conduct additional trainings as needed. If protocol modifications are significant, trial registries are updated.

Serious adverse events (e.g., participant death or hospitalization) for both mothers and/or children are reported on a real-time basis to the JHBSPH IRB. All study investigators have been trained and certified in basic human subject research, HIPAA compliance, and Social and Behavioral Research Best Practices for Clinical Research. All home visitors have also been trained and certified in HIPAA compliance.

\section{Standard Family Spirit}

Mothers at the control sites receive Family Spirit as usual, per existing ITC of MI program policies and procedures. In the standard of care, families receive regular home visits, delivered in the same sequence until 3 years postpartum (63 lessons total). Because the duration of enrollment for this pilot study is from pregnancy to 12 months postpartum, Standard Family Spirit participants should receive 47 lessons total. Home visitors at Standard Family Spirit sites use the Care4 platform to track their clients. They receive notifications about high depression scores, risk of self-harm, and potential domestic violence issues, but they do not receive notifications about other risk categories (first-time mom, substance use, nutrition, sexual and reproductive health) that would inform tailoring sequences in the Precision Family Spirit implementation approach. Details on the rationale for selecting these risk categories have been described elsewhere $[9,16]$.

In both arms of the study, home visitors provide referrals and connections to community agencies and services as needed. Existing procedures are in place to link participants to assistance that is available in their area. Home visitors often act as navigators and advocates for the families they serve. In addition, participant transportation to and from doctor and clinic appointments is common and will continue for enrolled participants in each arm.

The study team recognizes that differences may be small regarding number of prescribed lessons between Precision Family Spirit and Standard Family Spirit participants, but implementation outcomes may be potentially detectable because we are measuring program participant acceptability, satisfaction, quality of relationship between home visitor and client, retention, and adherence.

\section{Implementation strategy: Precision Family Spirit}

Our selection of an implementation strategy is guided by the Proctor et al. 2009 conceptual model of implementation [17]. Home visiting models in practice struggle with low retention rates [3]. Thus, we used design modularity as an implementation strategy that would improve engagement and retention. The specification of our implementation strategy can be found in Table 1 [18].

This modularized approach to Family Spirit is called Precision Family Spirit. Precision Family Spirit was developed using mixed methods, including a secondary data analysis to identify meaningful subgroups that could inform how and when to tailor, and stakeholder workshops with program implementers (supervisors and home visitors) to identify core lessons, and lessons relevant to certain subgroups of clients $[9,16]$. It consists of five lesson "pathways" that have been identified through 
Table 1 Specification of Family Spirit implementation strategy

\begin{tabular}{ll}
\hline Domain & Strategy: modularization of an EBT \\
\hline Actor(s) & Program development team; Implementers \\
Action(s) & Generate appropriate lesson pathways; implement these using an electronic support platform (Care4) \\
Target(s) of the action & Home visitors implementing Family Spirit; Clients receiving Family Spirit \\
Temporality & During the entire delivery of the program \\
Dose & Continuous \\
Implementation outcomes & Penetration (engagement and retention) among eligible clients; Adherence to the active prescribed ingredients of \\
affected & Family Spirit \\
Justification & Research suggests that retention is low in home visiting programs and tailoring of the program improves \\
& engagement and retention. \\
\hline
\end{tabular}

Note: Guidelines for specification of the Family Spirit implementation strategy from Proctor et al. (2013)

formative work across Family Spirit's national network of stakeholders [9]. There is a core set of lessons that every mother receives. For this pilot study, that means all Precision Family Spirit participants should receive 25 lessons from pregnancy to 12 months postpartum (the duration of enrollment). She receives additional lessons based on her emergent needs identified through data collection at home visits and at each assessment timepoint (Additional file 1). Thus, a Precision Family Spirit participant could be scheduled to receive between 25 and 41 lessons from pregnancy to 12 months postpartum. For example, if a participant is a first-time mother, she (and her family) will receive 6 additional baby care lessons (covering basic topics such as diapering and how to dress a baby) that are included in the Standard Family Spirit curriculum but may not be relevant for experienced mothers with previous children.

The study uses the Care4 implementation platform, which is a secure, HIPAA-compliant web-based application designed to capture data for the purposes of program implementation and evaluation [19]. Care4 is configured to notify home visitors when a participant needs to be added to any additional lesson pathways. Precision Family Spirit participants are assessed at each lesson visit using a measurement-based care approach [20-22] to understand and respond to emergent needs and help home visitors better track how their clients are doing over time. These short assessments are estimated to take 1-2 min to complete at each visit. Lessons are delivered in the same format as Standard Family Spirit, by trained home visitors in the home of the participant or a private place of their choosing.

\section{Data collection time points}

Participant assessments include maternal self-reports using Care4 and an interview (Table 2). Self-reports are conducted in participants' homes, another private location, or via text/email on an as needed basis. Data is recorded on either a tablet, computer, or paper. If data is collected on paper, the home visitor enters the data into
Care4 upon her return to the office. In some cases, assessments are conducted via text or email, once the home visitor discusses on a case by case basis with the study management team. For example, if a home visit is cut short, assessments may be completed post-visit by the participant. A secure link is sent via text or email with instructions on how to complete and submit. All participants will be assessed at four timepoints: baseline, 2 months, 6 months, and 12 months postpartum. Each assessment timepoint is estimated to take participants between $30 \mathrm{~min}$ and $1 \mathrm{~h}$ to complete. In an effort to mitigate participant time burden, home visitors complete assessments with participants during already-scheduled visits, by scheduling a separate visit, or by emailing or sending links to participants via SMS to complete the forms on their own time. Precision Family Spirit participants may also be asked to participate in a phone interview at study midpoint (between 4.5 and 6.5 months postpartum) and study endpoint (between 11 and 13 months postpartum), adding an additional 15-20 min of participant time burden for each interview completed. The purpose of these interviews is to further contextualize data being collected as part of the quantitative assessment.

Participants receive a gift card upon completion of the following assessment timepoints: baseline, 2 months, 6 months, and 12 months postpartum. Total possible remuneration for participants in the study is $\$ 70$ in gift cards.

From study midpoint until the endpoint of the study timeline, home visitors will be asked to participate in focus group discussions (FGDs) by video conference. Each site will participate in up to 10 short FGDs. FGDs will be designed to take approximately $30 \mathrm{~min}$ to complete and will be scheduled during regular study site meetings. They will be recorded with permission and transcribed verbatim. Home visitors receive a $\$ 15$ gift card upon completion of each FGD. Total possible remuneration for home visitors is $\$ 150$ in gift cards. 
Table 2 List of hybrid trial objectives and evaluation measures

\begin{tabular}{|c|c|c|c|c|c|}
\hline \multirow[t]{2}{*}{ Pilot trial objectives } & \multirow[t]{2}{*}{ Instrument } & \multicolumn{4}{|c|}{ Timepoint } \\
\hline & & Baseline & $\begin{array}{l}2 \text { months } \\
\text { postpartum }\end{array}$ & $\begin{array}{l}6 \text { months } \\
\text { postpartum }\end{array}$ & $\begin{array}{l}12 \text { months } \\
\text { postpartum }\end{array}$ \\
\hline \multicolumn{6}{|l|}{ Baseline characteristics } \\
\hline N/A & Maternal demographics & $x$ & & & \\
\hline \multicolumn{6}{|l|}{ Primary objectives } \\
\hline \multirow[t]{2}{*}{ Acceptability } & Program acceptability & & & $x$ & $x$ \\
\hline & Qualitative interview** & & & $x$ & $x$ \\
\hline \multirow[t]{2}{*}{ Satisfaction } & Participant satisfaction & & & $x$ & $x$ \\
\hline & Qualitative interview** & & & $x$ & $x$ \\
\hline $\begin{array}{l}\text { Home visitor-participant } \\
\text { relationship }\end{array}$ & $\begin{array}{l}\text { Working alliance inventory: } \\
\text { 1. Client WAI } \\
\text { 2. Home visitor WAI }\end{array}$ & & $x$ & $x$ & $x$ \\
\hline Retention & \multirow{2}{*}{\multicolumn{5}{|c|}{ Participant tracking information collected throughout the duration of the pilot study }} \\
\hline Adherence & & & & & \\
\hline \multicolumn{6}{|l|}{ Secondary objectives } \\
\hline Maternal substance use & $\begin{array}{l}\text { Alcohol, Smoking and Substance Involvement } \\
\text { Screening Test }\end{array}$ & $x$ & $x$ & $x$ & $x$ \\
\hline Maternal depression & Edinburgh Postnatal Depression Scale & $x$ & $x$ & $x$ & $x$ \\
\hline Parenting feeding practices & Child Feeding Assessment & & $x$ & $x$ & $x$ \\
\hline Maternal parental knowledge & Parent Knowledge Assessment & $x$ & $x$ & $x$ & $x$ \\
\hline \multirow[t]{2}{*}{ Child development } & Ages and Stages: Social Emotional - $2^{*}$ & & $x$ & $x$ & $x$ \\
\hline & Ages and Stages Questionnaire, 3rd Edition* & & $x$ & $x$ & $x$ \\
\hline Quality of life & EURO-QOL & $x$ & $x$ & $x$ & $x$ \\
\hline Maternal self-efficacy & Parenting Locus of Control & & & $x$ & $x$ \\
\hline Maternal stress & Perceived Stress Scale 4 & $x$ & $x$ & $x$ & $x$ \\
\hline $\begin{array}{l}\text { Substance abuse/domestic } \\
\text { violence risk }\end{array}$ & $\begin{array}{l}\text { Institute for Health and Recovery Integrated } \\
\text { Screening Tool }\end{array}$ & $x$ & $x$ & $x$ & $x$ \\
\hline Client priority outcomes & Top problems & $x$ & $x$ & $x$ & $x$ \\
\hline
\end{tabular}

*These measures will not be administered as part of the Care4 data collection platform, as they are licensed by ITC of MI external to the Care4 platform ${ }^{* *}$ Qualitative interviews will only be conducted with intervention participants at midpoint and endpoint

\section{Outcomes}

Selected measures are being used to assess implementation and clinical outcomes (Table 2).

\section{Primary outcomes}

Our primary outcomes of interest include participant acceptability, satisfaction, quality of relationship between home visitor and client, retention, and adherence. Acceptability is measured from the perspective of the participant using a 15-item scale [16] administered at study midpoint and endpoint. Satisfaction is captured through a 34-item questionnaire used in the Family Spirit trials and adapted specifically for this study and administered at midpoint (around 6 months postpartum) and endpoint. All Precision Family Spirit participants are invited to participate in a short qualitative interview to further explore acceptability and satisfaction using an 11-item interview guide. In addition, we will explore home visitor acceptability and satisfaction through focus group discussions that follow a 9-item interview guide. The quality of the relationship between home visitor and client is captured using the short version of the Working Alliance Inventory (WAI) administered to both participant and home visitor. The WAI measures three domains of the relationship: goal alignment, task alignment, and bond. Retention is measured by comparing the number of participants who drop out in each study arm. Adherence is measured by comparing the percentage of retained participants in each study arm who completed their lessons as prescribed.

\section{Secondary outcomes}

As part of the Hybrid Type 3 approach, we also monitor specific maternal behavioral and mental health outcomes and child physical and social-emotional development outcomes.

Maternal substance use is measured at all assessment timepoints (baseline, 2, 6, and 12 months postpartum) 
using an adapted version of the World Health Organization's validated Alcohol, Smoking and Substance Involvement Screening Test (ASSIST) [23, 24]. The ASSI ST screens for all levels of problem or risky substance use (alcohol, illegal drugs, prescription drugs, and tobacco use). A risk score is provided for each substance, and scores are grouped into low, moderate, or high risk.

Maternal depression is measured at all timepoints (baseline, 2, 6, and 12 months postpartum) using the validated Edinburgh Postnatal Depression Scale (EPDS) [25]. The EPDS is a 10-item self-rating scale that was specifically designed for women who are pregnant or have just had a baby. However, it has also been shown to be an effective measure for general depression in the larger population [26].

Parent feeding practices are measured at 2, 6, and 12 months postpartum using an assessment developed by the study team. It is adapted from a child feeding assessment used in two other CAIH randomized trials in Southwest Native American communities [27, 28]. The assessment will be used to assess feeding practices, introduction of complementary feeding and introduction of sugar sweetened beverages among newborns and infants.

Maternal parenting knowledge is measured at all timepoints (baseline, 2, 6, and 12 months postpartum) using a 10-item assessment developed by the study team and tied to core Family Spirit curriculum content.

Other maternal outcomes are measured at all timepoints (baseline, 2, 6, and 12 months postpartum) using short screening tools or measures: overall quality of life is measured using the EuroQol [29], maternal selfefficacy is measured using the Parental Locus of Control scale [30], parental stress is measured using the Perceived Stress Scale 4 [31], substance abuse/domestic violence risk is measured using the Institute for Health and Recovery Integrated Screening Tool [32], and client priority outcomes are measured using the Top Problems measure [33]. Each measure included in this assessment is valid and reliable, with some having been shortened through a previous secondary data analysis using Item Response Theory Methods [34].

Child development is measured using the parentreport, validated Ages \& Stages Questionnaire (ASQ-3) [35] and Ages \& Stages Questionnaire: Social-Emotional, Second Edition (ASQ:SE-2) [36]. The ASQ-3 is used to monitor all five domains of child development and screen children for developmental delays during the first 5 years of life. The ASQ:SE-2 focuses on socialemotional development of the child. The ITC of MI uses the ASQ-3 and ASQ:SE-2 as part of ongoing programmatic data collection. The CAIH will export sum scores at 2, 6, and 12 months postpartum for both assessments to use in data analysis.

\section{Quality assurance}

To ensure quality of participant consent procedures, all study staff have been certified to consent participants. Home visitors received initial in-person training on informed consent. Post-training, they had to demonstrate mastery of a full informed consent procedure with their supervisor prior to being certified. Program supervisors used a checklist to certify home visitors at their site.

Quality assurance of lesson delivery for both study arms occurs according to existing ITC of MI procedures. All home visitors are required to pass knowledge tests on each lesson prior to administering them to clients. Further, they must complete role plays and achieve a total average score of at least 3 out of 4 on a quality assurance form administered by a supervisor or program manager. To ensure ongoing high quality of lesson delivery, home visit observations are conducted at least biannually and ideally quarterly. For home visitors with at least 5 years of experience implementing Family Spirit, they are observed annually or more frequently if desired by the home visitor. Home visitors participating in this pilot trial are, in general, experienced in delivering Standard Family Spirit. Of 13 trained home visitors, 8 (62\%) have at least 5 years of experience as Family Spirit certified home visitors. Further, 11 (85\%) have at least 3 years of experience. All home visitors have been trained for at least a year before this pilot trial launched.

\section{Quality control of data management}

Assessment forms are initially checked for completion by the home visitor. All data are directly entered into the Care4 platform and stored in their secure electronic database. Weekly quality control procedures are carried out by the research coordinator to identify specific errors and missing data. The study statistician carries out quality control routines on an as needed basis in R. All $\mathrm{R}$ data sets are stored on a secure server. The research coordinator communicates all data queries to home visiting staff via email or on biweekly site calls.

\section{Sample size calculation and statistical analysis}

As this is a pilot study, our target sample $(N=60)$ was selected on the basis of feasibility of study completion, representativeness of the target study population (includes urban and rural sites) and with the desire to provide useful information to inform a fully powered research study (e.g., inclusion/exclusion criteria, variability in responses, estimated effects). Even so, with significance set to $a=0.05$ and power set to $80 \%$, using our $N$ $=60$ and accounting for $30 \%$ attrition, we would expect to be able to detect a large effect $(d=0.8)$ on continuous outcomes and a $35 \%$ difference in retention rates between the two groups. 


\section{Primary aim analysis}

As this is a pilot study, most data analysis will be exploratory, but based on best practices in the field to analyze clinical trial data. We will conduct an "intent to treat" analysis as the primary analysis approach. Baseline demographic characteristics will be explored to examine comparability between the groups. For continuous measures, we will explore average scores and distributions at each time point. To test for effects, we will use mixedeffects regression models (MRMs) to estimate average differences between outcomes on the standard vs. precision approaches using the scores on the acceptability, participant satisfaction, and home visitor-client relationship assessments. Specifically, we will add a dichotomous indicator for participants receiving the precision approach compared to the standard approach and examine the interaction of that dichotomous indicator with the change in outcome measure. The MRMs will include random effects at the individual and site levels to account for multiple time points within an individual and clustering by site. We will also examine the differences in proportion of participants who dropped out and who received $50 \%$ dosage using mixed effects logistic regression models accounting for clustering by site. For all analyses, we will report both unadjusted and adjusted analyses (adjustment based on differences in baseline demographics between the two groups at the $p<0.1$ level). For all qualitative data, we will use thematic analysis to analyze interview transcripts using Dedoose software [37]. As transcripts are reviewed, "memos" will be generated to document initial impressions of topics and themes. Segments of transcripts will then be assigned codes based on a priori (i.e., from the interview guide) or emergent themes (i.e., open coding) [38].

All analyses for the secondary outcomes will be done on an exploratory basis only.

\section{Discussion}

This is the first pilot randomized implementation trial of precision home visiting. We used the principals of design modularity to develop an implementation strategy to help guide home visitors in what to deliver and when, with the goal of improving retention and engagement while maintaining fidelity to what we know works. Our implementation strategy was developed through participatory methods with program implementers. This pilot implementation study will provide important preliminary understanding into the acceptability and feasibility of applying this precision approach to evidence-based home visiting, a major priority for the national home visiting field [5]. All evidence-based home visiting models incorporate content delivery into their implementation strategies, which is what this study focused on [13]. As such, authors see promise in translating findings and lessons learned from this pilot study into other models wishing to design precision approaches to home visiting.

\section{Limitations}

There are some limitations to this pilot study. First, we focused on content delivery (i.e., prescribed active ingredient) of the Family Spirit model. However, the home visitor-client relationship (e.g., unprescribed active ingredient) could be the focus of implementation strategies in home visiting [7, 39]. Results of data collected in this pilot trial through the Working Alliance Inventory will help us explore the quality of the home visitor-client alliance to understand whether tailoring content impacts this relationship.

Second, there was a small number of groups randomized into each study arm. Only four ITC of MI sites were included in this pilot trial. Therefore, we are unlikely to achieve completely comparable groups. However, within the context of these limitations, the results of this pilot trial can inform a fully powered cluster randomized trial in the future.

Third, there are some concerns regarding generalizability of study findings. Yet, a strength of this pilot study is that was designed for Native American communities, which are the most historically disenfranchised racial/ethnic subgroup in the US facing substantial behavioral health and sociodemographic disparities [40-42]. Positive findings in this population may provide promise for translation into other communities.

\section{Conclusion}

This pilot Hybrid Type 3 implementation study will provide a better understanding of how precision home visiting can be used to increase participant engagement and retention and provide insights as to whether outcomes can be strengthened by a precision approach. The results from this study can help refine the implementation and evaluation methodologies and inform a fully powered hybrid trial to test the effectiveness of a precision implementation strategy on improved participant retention and outcomes. Ultimately, this line of research will advance our understanding of how most efficiently and effectively to support the healthy growth and development of families with a wide range of needs.

\section{Supplementary Information}

The online version contains supplementary material available at https://doi. org/10.1186/s40814-020-00753-4.

Additional file 1:. Precision Family Spirit Lesson Pathways. Title of data: Precision Family Spirit Lesson Pathways - Pregnancy to 12 Months Postpartum. Description of data: Details of the five Precision Family Spirit pathways (pregnancy-12 months postpartum), including period and timepoint 
Additional file 2:. SPIRIT-CONSORT Extension to Pilot Trials. Title of data: Adapted and combined SPIRIT 2013 Checklist and CONSORT Extension to Pilot Trials. Description of data: An adaptation of recommended items to address in a clinical trial protocol and related documents

\section{Abbreviations}

AlHFS: American Indian Health and Family Services; ASQ-3: Ages and Stages Questionnaires; ASQ:SE-2: Ages \& Stages Questionnaire: Social-Emotional, Sec ond Edition; ASSIST: Alcohol, Smoking and Substance Involvement Screening Test; CAlH: Johns Hopkins Center for American Indian Health; EPDS: Edinburgh Postnatal Depression Scale; FGD: Focus group discussion; HomVEE: Home Visiting Evidence of Effectiveness; ITC of MI: Inter-Tribal Council of Michigan; JHBSPH IRB: Johns Hopkins Bloomberg School of Public Health Institutional Review Board; MIECHV: Maternal, Infant, and Early Childhood Home Visiting Program; MRM: Mixed-effects regression model; RCT: Randomized controlled trial; UIO: Urban Indian Organization; US: United States; WAI: Working Alliance Inventory

\section{Acknowledgements}

We respectfully acknowledge the mothers, children, and families who are participants in this study and all study team members, and the stakeholders who contributed time and wisdom to shaping the implementation strategy and research protocol

\section{Authors' contributions}

$\mathrm{Al}, \mathrm{EEH}, \mathrm{EK}, \mathrm{AL}, \mathrm{LM}$, and $\mathrm{AB}$ conceptualized the study design. Al, EEH, EK, AL, and $L M$ are responsible for the accuracy and security of data being collected. Outside investigators do not have access to study data. All authors contributed to the conceptualization of this paper. Al and EEH drafted the manuscript, and $A W, N N, E K, A L, L M$, and $A B$ substantively contributed to the background, study methods, and discussion sections. The authors reviewed and approved the final manuscript.

\section{Authors' information}

$\mathrm{Al}, \mathrm{EEH}, \mathrm{NN}$, and $\mathrm{AB}$ are members of a center that has a 35-year history of working with Native American communities in the Southwestern United States. This center is a part of the Johns Hopkins Bloomberg School of Public Health. AW is a faculty member at the Johns Hopkins Bloomberg School of Public Health and has expertise in conducting research in maternal, infant, and early childhood home visiting. The Precision Family Spirit Study Team and $E K, A L$, and $L M$ are members of a tribal consortium that has a 50-year history connecting member tribes and advocating and providing technical assistance to member tribes for programs and policies that enhance the health and well-being of Michigan tribes.

\section{Funding}

The primary funder for this grant titled Piloting a Precision Approach to Home Visiting is the Annie E. Casey Foundation (217.2100; Dr. Cynthia Weaver, Project Officer, 410-547-3660). The opinions or views expressed in this paper are those of the authors and do not necessarily represent the official position of the study sponsor. This funding source had no role in the study design and does not have any role in collection, management, analysis, and interpretation of data. Further, they have no role in the writing of this manuscript and the decision to submit the report for publication.

\section{Availability of data and materials}

Not applicable

\section{Ethics approval and consent to participate}

This trial has received approval from the funding agency and the Johns Hopkins Bloomberg School of Public Health Institutional Review Board (FWA\#0000287). The trial was approved by the participating tribal communities' governing bodies. All participants will complete written informed consent to participate; youth under age 18 will have written parent/guardian consent.

\section{Consent for publication}

Not applicable

\section{Competing interests}

The authors declare that they have no competing interests.

\section{Author details}

${ }^{1}$ Center for American Indian Health, Department of International Health, Johns Hopkins Bloomberg School of Public Health, Baltimore, MD, USA ${ }^{2}$ Maternal, Infant and Early Childhood Services, Inter-Tribal Council of Michigan, Inc., Sault Sainte Marie, MI, USA. ${ }^{3}$ Department of Population, Family and Reproductive Health, Johns Hopkins Bloomberg School of Public Health, Baltimore, MD, USA.

Received: 19 November 2019 Accepted: 16 December 2020

Published online: 06 January 2021

\section{References}

1. Mathematica. Home Visiting Evidence of Effectiveness Review: Executive Summary. 2019;OPRE Report \#2019-93.

2. Office of Planning, Research, \& Evaluation. Home Visiting Models Reviewing the Evidence of Effectiveness. 2020;OPRE Report \#2020-126.

3. Michalopoulos C, Shea Crowne S, Portilla XA, Lee H, Filene JH, Duggan A, et al. A Summary of Results from the MIHOPE and MIHOPE-Strong Start Studies of Evidence-Based Home Visiting; 2019. p. 2019-09.

4. Supplee LH, Duggan A. Innovative Research Methods to Advance Precision in Home Visiting for More Efficient and Effective Programs. Child Development Perspectives. 2019;0(0):1.

5. Supplee LH, Parekh J, Johnson M. Principles of Precision Prevention Science for Improving Recruitment and Retention of Participants. Prevention Science. 2018 Jul:19(5):689-94.

6. O'Brien RA, Moritz P, Luckey DW, McClatchey MW, Ingoldsby EM, Olds DL. Mixed methods analysis of participant attrition in the nurse-family partnership. Prevention Science. 2012;13(3):219-28.

7. Ingoldsby EM, Baca P, McClatchey MW, Luckey DW, Ramsey MO, Loch JM, et al. Quasi-experimental pilot study of intervention to increase participant retention and completed home visits in the nurse-family partnership. Prevention Science. 2013;14(6):525.

8. Ammerman RT. Commentary: Toward the Next Generation of Home Visiting Programs-New Developments and Promising Directions, Current Problems in Pediatric and Adolescent Health Care. 2016:46(4):126-9.

9. Haroz EE, Ingalls A, Wadlin J, Kee C, Begay M, Neault N, et al. Utilizing broad-based partnerships to design a precision approach to implementing evidence-based home visiting. J Community Psychology. 2020:1-14.

10. Barlow A, Mullany B, Neault N, Compton S, Carter A, Hastings R, et al. Effect of a paraprofessional home-visiting intervention on American Indian teen mothers' and infants' behavioral risks: a randomized controlled trial. American Journal of Psychiatry. 2013 Jan;170(1):83-93.

11. Barlow A, Varipatis-Baker E, Speakman K, Ginsburg G, Friberg I, Goklish N, et al. Home-visiting intervention to improve child care among American Indian adolescent mothers: a randomized trial. Archives of Pediatrics and Adolescent Medicine. 2006 Nov;160(11):1101-7.

12. Walkup JT, Barlow A, Mullany BC, Pan W, Goklish N, Hasting R, et al. Randomized controlled trial of a paraprofessional-delivered in-home intervention for young reservation-based American Indian mothers. Journal of the American Academy of Child \& Adolescent Psychiatry. 2009 Jun;48(6): 591-601.

13. Lyon AR, Lau AS, McCauley E, Vander Stoep A, Chorpita BF. A case for modular design: Implications for implementing evidence-based interventions with culturally diverse youth. Professional Psychology: Research and Practice. 2014;45(1):57.

14. Curran GM, Bauer M, Mittman B, Pyne JM, Stetler C. Effectivenessimplementation hybrid designs: combining elements of clinical effectiveness and implementation research to enhance public health impact. Medical Care. 2012;50(3):217.

15. Barlow A, Mullany B, Neault N, Goklish N, Billy T, Hastings $R$, et al. Paraprofessional-delivered home-visiting intervention for American Indian teen mothers and children: 3-year outcomes from a randomized controlled trial. American Journal of Psychiatry. 2015 Feb 1;172(2):154-62.

16. Haroz EE, Ingalls A, Kee C, Goklish N, Neault N, Begay M, et al. Informing precision home-visiting: Identifying meaningful subgroups of families who benefit most from Family Spirit. Prevention Science. 2019.

17. Proctor EK, Landsverk K, Aarons G, Chambers D, Glisson C, Mittman B. Implementation research in mental health services: an emerging science 
with conceptual, methodological, and training challenges. Administration and Policy in Mental Health. 2009;36(1):24

18. Proctor EK, Powell BJ, McMillen JC. Implementation strategies: recommendations for specifying and reporting. Implementation Science. 2013;8(1):139.

19. Care4Software I. Care4. 2019; Available at: care4soft.net.

20. Bickman L, Kelley SD, Breda C, de Andrade AR, Riemer M. Effects of Routine Feedback to Clinicians on Mental Health Outcomes of Youths: Results of a Randomized Trial. Psychiatric Services. 2011;62(12):1423.

21. Bickman L, Kelley SD, Athay M. The technology of measurement feedback systems. Couple and Family Psychology: Research and Practice. 2013;1(4): 274.

22. Scott K, Lewis CC. Using measurement-based care to enhance any treatment. Cognitive and Behavioral Practice. 2015;22(1):49.

23. WHO ASSIST Working Group. The Alcohol, Smoking and Substance Involvement Screening Test (ASSIST): development, reliability and feasibility. Addiction. 2002 Sep;97(9):1183-94.

24. Humeniuk R, Ali R, Babor TF, Farrell M, Formigoni ML, Jittiwutikarn J, et al. Validation of the Alcohol, Smoking And Substance Involvement Screening Test (ASSIST). Addiction. 2008;103(6):1039-47.

25. Cox JL, Holden JM, Sagovsky R. Detection of Postnatal Depression: Development of the 10-item Edinburgh Postnatal Depression Scale. British Journal of Psychiatry. 1987:150:782.

26. Kozinszky Z, Dudas RB. Validation studies of the Edinburgh Postnatal Depression Scale for the antenatal period. Journal of Affective Disorders. 2015;176:95.

27. Ingalls A, Rosenstock S, Foy Cuddy R, Neault N, Yessilth S, Goklish N, et al. Family Spirit Nurture (FSN) - a randomized controlled trial to prevent early childhood obesity in American Indian populations: trial rationale and study protocol. BMC Obesity. 2019;6:18.

28. Hedrick VE, Savla J, Comber DL, Flack KD, Estabrooks PA, Nsiah-Kumi PA, et al. Development of a brief questionnaire to assess habitual beverage intake (BEVQ-15): sugar-sweetened beverages and total beverage energy intake. Journal of the Academy of Nutrition and Dietetics. 2012 Jun;112(6): 840-9.

29. William A. EuroQol-a new facility for the measurement of health-related quality of life. The EuroQol Group. Health Policy. 1990;16(3):199.

30. Campis LK, Lyman RD, Prentice-Dunn S. The parental locus of control scale: Development and validation. Journal of Clinical Child Psychology. 1986; 15(3):260.

31. Cohen S, Kamarck T, Mermelstein R. A global measure of perceived stress. Journal of Health and Social Behavior. 1983 Dec;24(4):385-96.

32. Kennedy C, Finkelstein N, Hutchins E, Mahoney J. Improving Screening for Alcohol Use During Pregnancy: The Massachusetts ASAP Program. Maternal and Child Health Journal. 2004;8(3):137.

33. Weisz JR, Chorpita BF, Frye A, Ng MY, Lau N, Bearman SK, et al. Youth top problems: Using idiographic, consumer-guided assessment to identify treatment needs and to track change during psychotherapy. Journal of Consulting and Clinical Psychology. 2011;79(3):369-80.

34. Haroz EE. Pioneering the Home-Visiting Field: Precision-based approach to Family Spirit delivery. Annie E Casey Foundation 2017;Unpublished internal report.

35. Squires J, Bricker D. Ages \& Stages Questionnaires ${ }^{\circledast}$, Third Edition (ASQ ${ }^{\circledast}-3$ ): A Parent-Completed Child Monitoring System. Baltimore: Paul H. Brookes Publishing Co., Inc.; 2009.

36. Squires J, Bricker D, Twombly E. Ages \& Stages Questionnaires ${ }^{\oplus}$ : SocialEmotional, Second Edition (ASQ ${ }^{\otimes}$ SE-2): A Parent-Completed Child Monitoring System for Social-Emotional Behaviors. Baltimore: Paul H. Brookes Publishing Co., Inc.; 2015.

37. Lieber E. Weisner T. Dedoose: Taylor J; 2011.

38. Corbin A, Strauss A. Basics of qualitative research: Techniques and procedures for developing grounded theory. Fourth ed. Thousand Oaks: SAGE Publications, Inc:; 2015

39. Burrell L, Crowne S, Ojo K, Snead R, Oâ€而Neill K, Cluxton-Keller F, et al. Mother and Home Visitor Emotional Well-Being and Alignment on Goals for Home Visiting as Factors for Program Engagement. Maternal and Child Health Jouranl. 2018 10/01;22(1):43-51.

40. Indian Health Service. Report on Behavioral Health 2018 Edition. 2018.

41. Indian Health Service. Trends in Indian Health 2014 Edition. 2015.

42. Indian Health Service. Indian Health Disparities. 2019; Available at: https:// www.ihs.gov/newsroom/factsheets/disparities/. Accessed April 4, 2020.

\section{Publisher's Note}

Springer Nature remains neutral with regard to jurisdictional claims in published maps and institutional affiliations.

\section{Ready to submit your research? Choose BMC and benefit from:}

- fast, convenient online submission

- thorough peer review by experienced researchers in your field

- rapid publication on acceptance

- support for research data, including large and complex data types

- gold Open Access which fosters wider collaboration and increased citations

- maximum visibility for your research: over $100 \mathrm{M}$ website views per year

At BMC, research is always in progress.

Learn more biomedcentral.com/submissions 